\title{
Experimental Searches for Non-Baryonic Dark Matter: WIMP Direct Detection*
}

\author{
Angel Morales ${ }^{\dagger}$ \\ Laboratory of Nuclear and High Energy Physics and Canfranc Underground Laboratory (LSC) \\ University of Zaragoza \\ 50009 Zaragoza. Spain
}

An overview of the current status of WIMP direct searches is presented, emphasizing strategies, achievements and prospects.

\section{Introduction}

Experimental observations and robust theoretical arguments have established that our universe is essentially non-visible, the luminous matter scarcely accounting for one per cent of the energy density of a flat universe. The distribution of a flat universe $\left(\Omega=\Omega_{M}+\Omega_{\Lambda}=1\right)$ attributes to the dark energy about $\Omega_{\Lambda} \sim 70 \%$, whereas the matter density takes the remaining $\Omega_{M} \sim 30 \%$, consisting of, both, visible $\left(\Omega_{l} \sim 0.5 \%-1 \%\right)$ and non-visible (dark) matter. This dark component consists of ordinary baryonic matter $\left(\Omega_{B} \sim\right.$ $4-5 \%$ ), (possibly made by machos, jupiters, dust, black holes, etc.) and a large fraction (up to $\Omega_{N B} \sim 25 \%$ ) of non baryonic dark matter, supposedly made by non-conventional, exotic particles. The minimal requirements to be fulfilled by the non-baryonic dark particles are to provide the right relic abundance, to have non-zero mass, zero electric charge and a weak interaction with ordinary matter. There are several candidates to such species of matter provided by schemes beyond the Standard Model of Particle Physics. The galactic DM axions, the SUSY WIMPs (like neutralinos) and the light neutrinos (of some non standard models) are particularly attractive. The WIMPs, Weak Interacting Massive Particles, are favorite of experimentalists $(\sim 20$ experiments for WIMPs vs. 2 for axions) and so of this talk. This talk deal

${ }^{*}$ Invited Review Talk given at the VII Workshop on Topics in Astroparticle and Underground Physics (TAUP2001), Sept.2001, LN Gran Sasso, Italy

†amorales@posta.unizar.es with the recent efforts done in its direct search illustrated by a few, selected experiments.

Galactic halo WIMPs could be directly detected by measuring the nuclear recoil produced by their elastic scattering off target nuclei in suitable detectors at a rate which depends of the type of WIMP and interaction. In the case of WIMPs of $m \sim G e V$ to $T e V$ and $v \sim 10^{-3} c$ the nuclear recoil in the laboratory frame $E_{R}=\frac{\mu^{2}}{M} v^{2}(1-\cos \theta)$ is in the range from 1 to $100 \mathrm{KeV}$. $M$ is the nuclear mass, $\mu$ the $(m, M)$ reduced mass and $\theta$ the WIMP-nucleus (c. of m.) scattering angle. Only a fraction $Q E_{R}=E_{v i s}\left(\equiv E_{e e e}\right)$ of the recoil energy is visible in the detector, depending on the type of detector and target and on the mechanism of energy deposition. The so-called Quenching Factor Q is essentially unit in thermal detectors whereas for the nuclei used in conventional detectors it ranges from about 0.1 to 0.6 . The energy delivered by the WIMP results in a small signal (1-100 KeV) which shows up even smaller (for $\mathrm{Q}<1$ ). Moreover this signal falls in the low energy region of the spectrum, where the radioactive and environmental background accumulate at much faster rate and with similar shape. That makes WIMP signal and background practically undistinguishable. On the other hand, the smallness of the neutralino-matter interaction cross-section implies that the process looked for is very rare.

Customarily, one compares the predicted event rate with the observed spectrum. If the former turns out to be larger than the measured one, the particle which would produce such event rate can be ruled out as a Dark Matter candidate. That is 
expressed as a contour line $\sigma(\mathrm{m})$ in the plane of the WIMP-nucleon elastic scattering cross section versus the WIMP mass. That excludes, for each mass $\mathrm{m}$, those particles with a cross-section above the contour line $\sigma(\mathrm{m})$. The level of background sets, consequently, the sensitivity of the experiment in eliminating candidates or in constraining their masses and cross sections.

However, this simple comparison will not be able to identify the WIMP. A convincing proof of its detection should be provided by a distinctive signature characteristic of WIMPs. Such distinctive labels do exist: they are originated by the motion of the Earth in the galactic halo [1]:2]. These signatures are an annual modulation of the rate and a directional asymmetry of the nuclear recoil. Narrowing first the window of the possible WIMP existence and looking then for its identification is the purpose of the experimental searches. Table 1 gives an overview of the experiments on direct detection of WIMPs currently in operation or in preparation, which is the subject of this talk. General reviews for WIMP dark matter are given in Ref. [3]. WIMP direct detection is reviewed, for instance in Ref. [4, [5].

WIMPs can be also looked for, indirectly, in the galactic halo, looking for its presence in cosmic ray experiments in terms of antiprotons, positrons or gamma rays produced by WIMP annihilation in the halo. One can also search in underground, underwater or under-ice detectors, looking (also indirectly) for WIMPs through the high energy neutrinos emerging as final products of the WIMP annihilation in celestial bodies (Earth or Sun).

\section{Strategies for WIMP direct detection}

In order to plan an experiment it is essential to know how small the WIMP-nucleus interaction rates are, to estimate which are the chances of a positive result, for a given experimental sensitivity. According to the SUSY model employed and the choice of the various parameters, the predicted rate encompass various orders of magnitude, going from $R \sim 1-10 \mathrm{c} / \mathrm{Kg}$ day down to $10^{-4}-10^{-5} c / K g d a y$. The $\sigma_{\chi N}$ calculations are made within the Minimal Supersymmetric ex- tension of the Standard Model, MSSM, as basic frame, implemented in various schemes (see Ref.[6]). Besides the peculiarities of the SUSY model there is a wide choice of parameters entering in the calculation of the rates: the halo model, the values of the WIMP velocity distribution parameters, the three levels of the WIMPnucleus interaction (quark-nucleon-nucleus) and the constraint of getting the proper relic abundance of the candidates. The theoretical prediction of the rates are presented as "scatter plots" extending along the various orders of magnitude quoted above. Some of the most favourable predictions are already testable by the leading experiments which have, in fact, penetrate into the scatter plot of predictions. The bottom of the plot is still far away of the detector sensitivity. It should be noted, however, that most of the experimental searches for SUSY-WIMP's concentrate in the dominant, coherent interaction, which provides the largest signals.

How the WIMP direct search proceeds? To face the challenge of detecting signals of small size $\left(E_{R}<100 \mathrm{KeV}\right)$ produced at a very small rate $\left(R \sim 10 \rightarrow 10^{-5} \mathrm{c} / \mathrm{Kgday}\right.$ ) (equivalent to detecting processes of $\sigma \sim 10^{-10} p b$ (for a Ge detector)), which have a similar shape than the background, ultra-low background detectors and a radioactivity-free environment are needed. Example of low background recently achieved is the case of IGEX, which in its raw Ge background spectra have reached a level of $10^{-1} \sim$ $10^{-2} \mathrm{c} / \mathrm{KeVK}$ gday in the low energy region, and the cases of the CDMS and EDELWEISS experiments where their present total rate is of $\sim 1 \mathrm{c} /$ Kgday. Because of the small recoil energy delivered in the WIMP interaction, detectors of very low energy threshold and high efficiency are mandatory. That is the case for the bolometer experiments (MIBETA, CRESST, ROSEBUD, CUORICINO, CDMS and EDELWEISS) which seen efficiently the energy delivered by the WIMP (quenching factor is unity) and which have achieved very low energy thresholds $\left(E_{T H R} \sim\right.$ hundreds of $\left.e V\right)$. Large masses of targets are also recommended, to increase the probability of detection and the statistics. The DAMA, UKDMC and Zaragoza scintillations experiments 
use from 50 to $100 \mathrm{Kg}$ of NaI; the CUORICINO bolometer experiment plans to employ $42 \mathrm{Kg}$ of $\mathrm{TeO}_{2}$ crystals and the ZEPLIN detector which uses (according to the various versions) from 20 to $30 \mathrm{Kg}$ of Xe. It is remarkable that small size, first generation detectors have reached exclusions $\sigma_{\chi p}>10^{-5}-10^{-6} p b\left(10^{-41}-10^{-42} \mathrm{~cm}^{2}\right)$ in the range of masses relevant for SUSY-WIMPs.

To get an ultralow background, the radiopurity of the detector, components, shielding and environment must be the best achievable with the current state-of-the-art. The next step is to reject components of the background by discriminating electron recoils (tracers of the background) from nuclear recoils (originated by WIMPs and neutrons). Methods used to discriminate backgrounds from nuclear recoils are either simply statistical, like a Pulse Shape analysis (PSD), based on the different timing behaviour of both types of pulses, or event by event by measuring simultaneously, the ionization (or scintillation) and the heat, and noting that for a given deposited energy (measured as phonons) the recoiling nucleus ionizes less than the electrons. Examples of PSD are the sodium iodide experiments of UKDMC, Saclay, DAMA and ANAIS. Event by event discrimination has been successfully applied in CDMS and EDELWEISS by measuring ionization and heat and now in CRESST and ROSEBUD by measuring light and heat.

Another discriminating technique is that used in the two-phase liquid-gas Xenon detector with ionization plus scintillation, of the ZEPLIN series of detectors. An electric field prevents recombination, the charge being drifted to create a second pulse in addition to the primary pulse. The amplitudes of both pulses are different for nuclear recoils and electrons allowing their discrimination.

One could use instead threshold detectors-like neutron dosimeters-which are blind to most of the low Linear Energy Transfer (LET) radiation $(\mathrm{e}, \mu, \gamma)$ and so able to discriminate gamma background from neutrons (and so WIMPs). Detectors which use superheated droplets which vaporize into bubbles by the WIMP (or other high LET particles) energy deposition are those of the SIMPLE and PICASSO experiments. An ultimate discrimination will be the identification of the different kind of particles by the tracking they left in, say, a TPC, plus the identification of the WIMP through the directional sensitivity of the device (DRIFT).

\section{Excluding WIMPs which cannot be dark matter}

How to proceed to exclude a WIMP candidate? The direct experiments measure the differential event rate (energy spectrum) in the customary differential rate unit (dru) $\left[\frac{d R}{d E_{\mathrm{VIS}}}\right]^{\exp }(c / K e V K g d a y) . \quad$ The registered counts $\mathrm{R}$ contain the signal and the background. Then, by applying discrimination techniques one disentangle at least partially the nuclear recoils from the background events. The resulting residual rate is then compared (in terms of $\mathrm{m}$, and $\left.\sigma_{\chi N}\right)$ with the theoretical nuclear recoil rate due to WIMPs interaction (in counts per $\mathrm{KeV} \mathrm{Kg}$ day)

$\left[\frac{d R}{d E_{\mathrm{VIS}}}\right]^{\mathrm{Th}}=7.76 \times 10^{14} \frac{N}{Q} \frac{\rho}{v_{E}} \frac{(m+M)^{2}}{4 m^{3} M} F^{2} \sigma_{\chi N}^{S I} \tau$

(with $\sigma$ in $\mathrm{cm}^{2}$, $\mathrm{m}$ and $\mathrm{M}$ in GeVs, $v$ in $K m s^{-1}, \rho$ in $\mathrm{GeV} \mathrm{cm}^{-3}$ and $\mathrm{N}$ in $\mathrm{Kg}^{-1}$ ) where $\rho$ is the local density of WIMP, $\mathrm{N}$ the number of target nuclei, $F^{2}$ is the nuclear form factor, and $\tau\left(v_{\text {esc }} \rightarrow \infty\right)=$ $\operatorname{erf}(x+y)-\operatorname{erf}(x-y)$, with

$$
x, y=\sqrt{\frac{3}{2}}\left(v_{\min }, v_{E}\right) \frac{1}{v_{\mathrm{rms}}}, \quad v_{\mathrm{rms}} \sim \sqrt{\frac{3}{2}} v_{\mathrm{sun}}
$$

$v$ the WIMP velocity (Earth/Lab frame) and $v_{E}$ the velocity of Earth/Solar system with respect to the halo.

$v_{\text {min }}\left(E_{R}\right)=\frac{m+M}{m}\left(E_{R} / 2 m\right)^{\frac{1}{2}}$ is the minimal velocity to produce a recoil $E_{R}$. The spinindependent nuclear cross-section is usually normalized in terms of that on nucleons

$$
\sigma_{\chi N}^{S I}=A^{2} \frac{\mu_{\chi N}^{2}}{\mu_{\chi n}^{2}} \sigma_{\text {nucleon(p.n) }}^{\text {scalar }}
$$

Those values of $(\sigma, \mathrm{m})$ predicting a recoil spectrum above the observed rate

$$
\left[\frac{d R}{d E_{\mathrm{VIS}}}\right]^{\mathrm{Th}} \geq\left[\frac{d R}{d E_{\mathrm{VIS}}}\right]_{\mathrm{UppBound}}^{\mathrm{exp}}
$$


are excluded. The region above the contour $\sigma(m)$ is depicted as an exclusion plot of those WIMPs of mass $m$ with interaction cross-section above $\sigma$. Obviously, the smaller the background the better the exclusion. However excluding a WIMP is not enough.

\section{The identification of WIMP dark mat- ter}

After reducing maximally the background and extremating the discrimination $(99.99 \%)$ of the detector, one should look for asymmetries characteristic of WIMP signals. Typical smoking guns of WIMPs would be the annual modulation of the rate [1], the forward/backward asymmetry of the nuclear recoil [2] or the nuclear target dependence of the rates [7].

The two kinematical asymmetries characteristic of WIMPs signals are originated by the Earth motion through the galactic halo. The Earth orbital motion around the Sun has a summer/winter variation, which produces a small annual modulation of the WIMP interaction rates, of the order $O\left(\frac{v_{\mathrm{rot}, \mathrm{E}}}{v_{h}}\right) \sim \frac{15}{270} \sim 5 \%$ [1]. The observation of this small modulation of a very small signal requires large target mass and exposure, superbe stability and extreme control of systematics and of other stational effects.

The orbital velocity of Earth around the sun is of $30 \mathrm{Kms}^{-1}$ in an orbit inclined $\alpha=60^{\circ}$ with respect to the galactic disk

$$
\begin{gathered}
v_{E, r}=30 \cos \alpha \cos \omega\left(t-t_{0}\right) \rightarrow 15 \cos \omega\left(t-t_{0}\right) K m s^{-1} \\
\omega=\frac{2 \pi}{T} \quad T=1 \text { year } \quad \mathrm{t}_{0}: \text { June } 2^{\text {nd }}
\end{gathered}
$$

So, the velocity of the Earth (and of our earthborne detector) relative to the galactic halo is

$$
v_{E}=v_{\text {sun }}+15 \cos \omega\left(t-t_{0}\right) K m s^{-1}
$$

Consequently, in summer there is a component of the Earth' motion around the sun parallel to the sun motion through the galaxy which adds $15 \mathrm{Kms}^{-1}$. On the contrary, in winter the same occurs but the motion is antiparallel and so one has to subtract $15 \mathrm{Kms}^{-1}$. The result is that the detector moves slightly faster in June than in December ( $5 \%$ effect), and consequently a modulation of the WIMP interaction rates follows, given at first order by

$$
S(t)=S_{0}+S_{m} \cos \omega\left(t-t_{0}\right)[+B]
$$

where $S_{0}$ is the average signal amplitude, $S_{m}$ the modulated amplitude and $B$ the constant background.

A second characteristic signature of the WIMP is provided by the directional asymmetry of the recoiling nucleus [2]. The WIMPs velocity distribution in the Earth frame is peaked in the opposite direction of the Earth/Sun motion through the halo, and so the distribution of nuclear recoils direction shows a large asymmetry forward/backward (F/B) not easily mimicked by the supposedly isotropic background. The order of magnitude of the effect is large because the solar system' motion around the galactic center $v_{\text {sun }}$, and the typical WIMP velocity in the halo, $v_{h}$, are of the same order $O\left(\frac{v_{\text {sun }}}{v_{h}}\right) \sim \frac{230}{270} \sim 1$.

The angular dependence of event rate is given by (see Ref.[2])

$$
\frac{d^{2} R}{d E_{R} d(\cos \gamma)}=
$$

$$
\frac{N \rho \sigma}{\sqrt{\pi}} \frac{(m+M)^{2}}{2 m^{3} M v_{\text {halo }}} \exp \left\{-\frac{\left(v_{\mathrm{E}} \cos \gamma-v_{\min }\right)^{2}}{v_{\mathrm{h}}^{2}}\right\}
$$

where $\gamma$ is the angle of the recoiling nucleus.

Recently, the nuclear recoil angular dependence of WIMP interactions has been analyzed in different halo models with the purpose of exploring how well a directional signal can be distinguished without ambiguity from the background -with independence of the halo model-. Quite remarkably if the device has angular resolution sensitivity, few events will be enough to distinguish the signal, and not too many are needed if it has only F/B sensitivity. See Ref.[8].

Another asymmetry is the nuclear target dependence of the rate [7]. However, due to the differences in the intrinsic backgrounds of the various targets, it is not easy to get reliable conclusions. Some experiments are operating (or can 


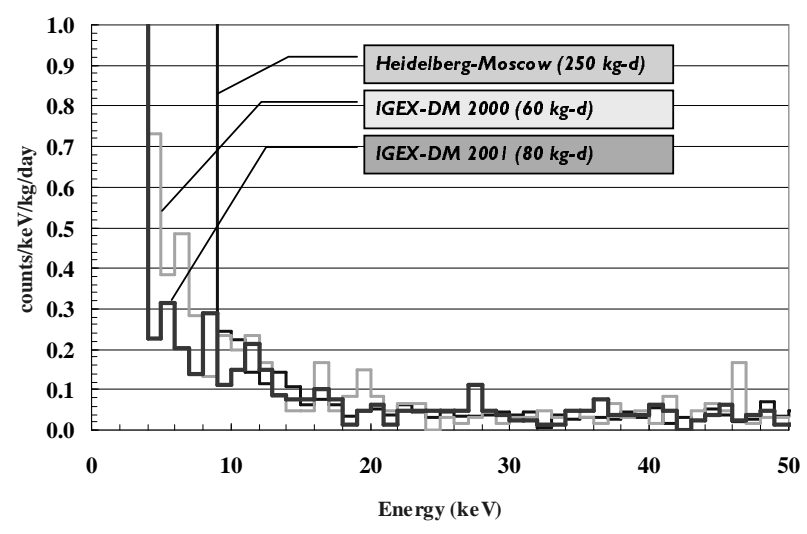

Figure 1.

do it) sets of similarly produced crystals of different nuclear targets in the same environment like ROSEBUD $\left(\mathrm{Ge} / \mathrm{Al}_{2} \mathrm{O}_{3} / \mathrm{CaWO}_{4}\right)$.

\section{Germanium Experiments}

The high radiopurity and low background reached in Germanium detectors, the low energy threshold which can be obtained, their reasonable quenching factor (about 25\%) and other nuclear merits make Germanium a good option to search for WIMPs with detectors and techniques fully mastered [9].

The International Germanium Experiment (IGEX) 10 is using one enriched detector of ${ }^{76} \mathrm{Ge}$ of $\sim 2.1 \mathrm{Kg}$ to look for WIMPs in the Canfranc Underground Laboratory. It has an energy threshold of $4 \mathrm{keV}$ and an energy resolution of 0.8 $\mathrm{keV}$ at the $75 \mathrm{keV} \mathrm{Pb} \mathrm{x}$-ray line. The detector is fitted in a cubic block in lead being surrounded by not less than 40-45 cm of lead of which the innermost $25 \mathrm{~cm}$ are archaeological. A muon veto and a neutron shielding of $40 \mathrm{~cm}$ of polyethylene and borated water completed the set-up [11, 12]. The spectrum of IGEX-2001 12 together with that of a previous run [1] (IGEX-2000) are shown in Fig. 11 in comparison with that of the HeidelbergMoscow experiment. The $\mathrm{H} / \mathrm{M}$ experiment[13] is another enriched-Ge experiment (energy threshold of $9 \mathrm{KeV}$ ), already completed, which has been

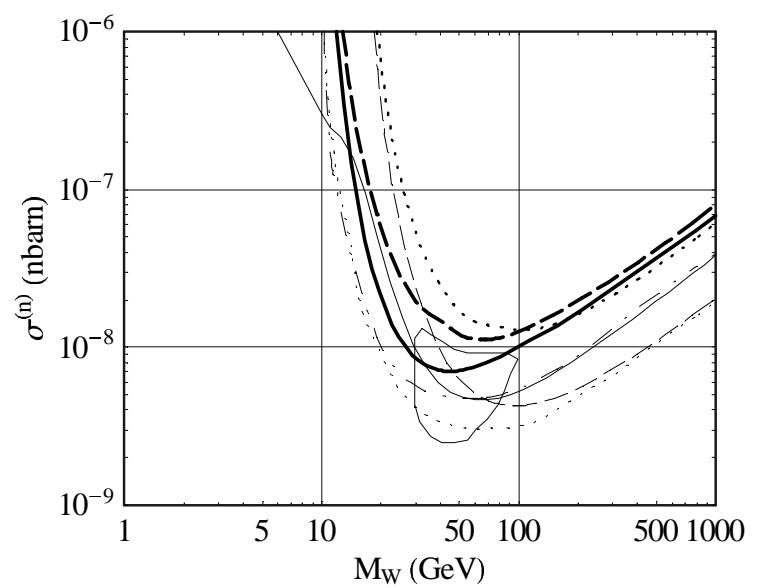

Figure 2.

running at Gran Sasso.

The exclusion plots derived for these three Ge experiments are depicted in Fig. 2 following the same method: IGEX-2001 (thick solid line), IGEX-2000 (thick dashed line) and HeidelbergMoscow (thick dotted line). IGEX-2001 improves the exclusion of all other Ge-ionization experiments for a mass range from $20 \mathrm{GeV}$ up to $200 \mathrm{GeV}$, which encompass the DAMA mass region [14. In particular, this new IGEX result excludes WIMP-nucleon cross-sections above 7 $\times 10^{-6} \mathrm{pb}$ for masses of $40-60 \mathrm{GeV}$ and enters the DAMA region excluding the upper left part of this region. That is the first time that a direct search experiment with a Ge-diode without background discrimination, but with very low (raw) background, enters such region. A further $50 \%$ background reduction between $4 \mathrm{keV}$ and $10 \mathrm{keV}$ (which could be reasonably expected) would allow IGEX to explore practically all the DAMA region in $1 \mathrm{~kg}$ y of exposure. (We refer to the Igor G. Irastorza contribution to these Proceedings and Ref.[9.12]).

Also shown for comparison are the contour lines of the other experiments, CDMS[15] and EDELWEISS 16 (thin dashed line), which have entered that region by using bolometers which also mea- 
sure ionization and which will be described later on. The DAMA region (closed line) corresponding to the annual modulation effect reported by that experiment 14] and the exclusion plot obtained by DAMA NaI-0 [17] (thin solid line) by using statistical pulse shape discrimination are also shown. In the CDMS case two contour lines have been depicted, the exclusion plot published in Ref.[15] (thin dotted line) and the CDMS expected sensitivity contour (thin dot-dashed line) (See B. Sadoulet contribution to these Proceedings.)

A new experiment GEDEON (GErmanium DEtectors in ONe cryostat) is planned for Canfranc [9]. It will use the technology developed for the IGEX experiment. The cell-unit will consist of a set of $\sim 1 \mathrm{~kg}$ germanium crystals, of a total mass of about $28 \mathrm{~kg}$, placed together in a compact structure inside one only cryostat. Expected backgrounds are $\sim 10^{-3} \mathrm{c} / \mathrm{KeVK}$ gday in the low energy region. A total mass of $\sim 112 \mathrm{Kg}$ of $\mathrm{Ge}$ (or 4 cells) is projected for a second phase of GEDEON.

Two more Ge experiments running or in preparation, both in Gran Sasso, are that of the Heidelberg Dark Matter Search (HDMS) and the GENIUS-Test Facility. The small detector of HDMS has achieved a background still higher than that of $\mathrm{H} / \mathrm{M}$ and so the results will not be include here. See Ref. 18]. Most of the attention of this Collaboration goes now to the preparation of a small version (GENIUS-Test Facility) of the GENIUS project [19]. GENIUS is a multipurpose detector, consisting of enriched Ge detectors of about $2 \frac{1}{2} \mathrm{Kg}$ each (up to a total of 0.1 to 10 tons) which uses the novel idea of immerse the crystals directly into a large tank of liquid nitrogen. GENIUS-TF (fourteen detectors of natural isotopic abundance and low nominal energy threshold), now in preparation, is intended to test the GENIUS project and at the same time to search for WIMP. (We refer to the H.V. Klapdor contribution to these Proceedings for details).

\section{WIMP searches with $\mathrm{NaI}$ scintillators}

The sodium iodide detectors are very attractive devices to look for WIMP. Both nuclei have

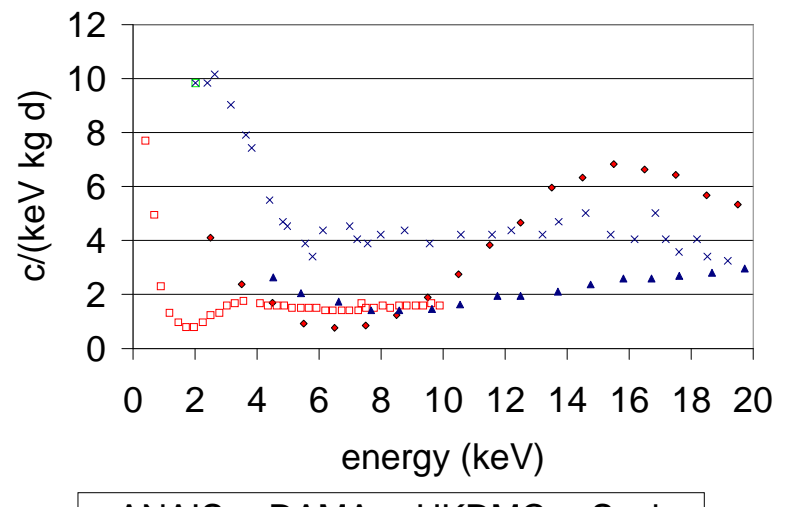

- ANAIS DAMA $\cdot$ UKDMC $\times$ Saclay

Figure 3 .

non-zero spin $\left({ }^{23} \mathrm{Na} J=\frac{3}{2},{ }^{127} I J=\frac{5}{2}\right)$ and then sensitive also to spin dependent interaction. Iodine is a heavy nucleus favourable for spinindependent interactions. The quenching factor is small $(Q<10 \%)$ for $\mathrm{I}$, and medium for $\mathrm{Na}$ $(Q \sim 30-40 \%)$. Backgrounds lesser than or of the order of $\sim 1$ count per $\mathrm{KeV} \mathrm{Kg}$ day in the few $\mathrm{KeV}$ region have been achieved. There exists four NaI experiments running: DAMA, UKDMC, (in various detectors and projects), ELEGANTS and ANAIS.

The NaI scintillators can be endowed with Pulse Shape Discrimination (PSD) to distinguish statistically gamma background from WIMPs (or neutron) signals, because of the different timing behaviour of their pulses. From such statistical analysis it results that only a few percent (depending on the energy) of the measured background can be due to nuclear recoils. The background spectra (before PSD) of the four NaI experiments ANAIS 20, DAMA 14], UKDMC 21] and Saclay [22] are shown comparatively in Fig. 3 (1 to $2 c / K e V K g d a y$ in DAMA, UKDMC and ANAIS and of 2 to $10 \mathrm{c} / \mathrm{KeVKgday}$ in Saclay and ELEGANTS [23]).

The United Kingdom Dark Matter Collaboration (UKDMC) uses radiopure $\mathrm{NaI}$ crystals of various masses (2 to $10 \mathrm{~kg}$ ) in various shielding conditions (water, lead, copper) in Boulby 


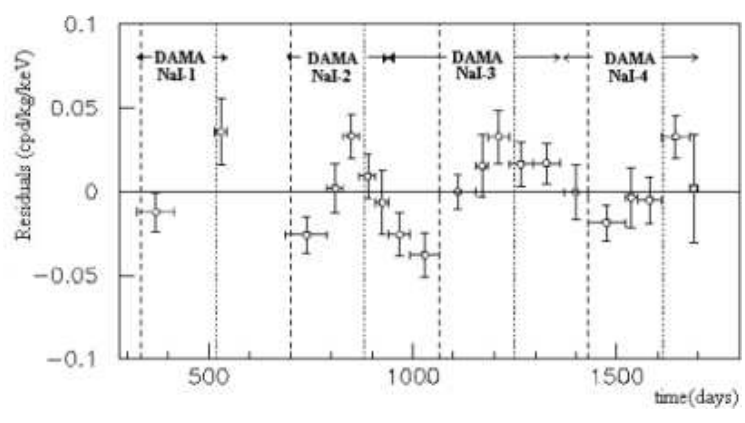

Figure 4.

21. Typical thresholds of $4 \mathrm{keV}$ have been obtained. Results from NaI crystals of various dimension showed a small population ("anomalous") of pulses of an average time constant shorter than that of gamma events and attributed probably to an alpha surface contamination. New crystals with polished surfaces (and operating unencapsulated in dry nitrogen atmosphere) do not show such anomaly. UKDMC is also preparing, NAIAD (NaI Advanced Detector) wich will consist of 50-100 kg in a set of unencapsulated crystals to avoid surface problems and improve light collection. (See S. Hart contribution to these Proceedings.)

ANAIS (Annual Modulation with NaI's) will use $107 \mathrm{~kg}$ of $\mathrm{NaI}(\mathrm{Tl})$ in Canfranc. A prototype of one single crystal $(10.7 \mathrm{~kg})$ is being developed. The components of the photomultiplier have been selected for its radiopurity. Pulse Shape analysis has been performed and the possible presence of "anomalous" events investigated. No evidence of such anomaly has been found. The preliminary results refer to an exposure of $1225.4 \mathrm{kgday}$. Fig. 3 shows the background after the noise rejection. The energy threshold is of $\sim 4 \mathrm{keV}$ and the background level registered from threshold up to $10 \mathrm{keV}$ is about $1.2 \mathrm{c} / \mathrm{KeVKgday}$. (We refer to the S. Cebrián contribution to these Proceedings).

The DAMA experiment 14] uses 9 radiopure $\mathrm{NaI}$ crystals of $9.7 \mathrm{Kg}$ each, viewed by two PMT. The background spectrum is shown also in Fig. 3.
The software energy threshold is at $E_{T h r}=2 \mathrm{keV}$ and the energy resolution at $2-5 \mathrm{keV}$ is $\Gamma \sim 2-$ $2.5 \mathrm{keV}$. The PSD method applied to the DAMA NaI-0 17] running lead to a background reduction of $85 \%$ (at $4-6 \mathrm{keV}$ ) and $97 \%$ (at $12-20 \mathrm{keV}$ ), providing exclusion plots which have surpassed that of germanium.

The main objective of DAMA is to search for the annual modulation of the WIMPs signal. Such modulation has been found and attributed by the Collaboration to a WIMP signal. After $57986 \mathrm{Kg}$ day of statistics the residuals of the rate vs time, looks as shown in Fig. It. It modulates according to $A \cos \left[\omega\left(t-t_{0}\right)\right]$ with period and phase consistent with 1 year and 2nd June, respectively. The probability of absence of modulation is $\sim 4 \times 10^{-4}$. The DAMA global results [14] (NaI, $1,2,3,4$ runnings) in the case of assuming the WIMP interpretation, lead to a WIMP of mass and cross-section given by $M_{W}=\left(52_{-8}^{+10}\right) \mathrm{GeV}$ $\xi \sigma^{p}=\left(7.2_{-0.9}^{+0.4}\right) \times 10^{-6} p b$. A maximum likelihood favours the hypothesis of presence of modulation with the above $M_{W}, \xi \sigma_{p}$ values at $4 \sigma$ C.L. The $(\sigma$, $\mathrm{m}$ ) region for spin independent coupled WIMP is the "triangle" zone depicted in Fig. 2. (We refer to the A. Incicchitti contribution to these Proceedings and Ref. 14]). An extension of DAMA up to $250 \mathrm{Kg}$ of NaI (LIBRA) is being prepared.

The DAMA results have aroused great interest and controversies. It is imperative to confirm the DAMA results by other independent experiments with NaI (like LIBRA, NAIAD, ANAIS, or ELEGANTS) and with other nuclear targets. For instance, CUORICINO, with $42 \mathrm{Kg}$ of $\mathrm{TeO}_{2}$, now being mounted. The DAMA $\sigma(\mathrm{m})$ region is being explored, also by the standard method followed for excluding WIMPs. Various experiments have already reached and (partially) excluded the DAMA $(\sigma, \mathrm{m})$ region. In particular CDMS, EDELWEISS and IGEX have derived exclusion contours which "enter" the DAMA region (see Fig. 2).

The OSAKA group is performing a search with the ELEGANTS $\mathrm{V}$ NaI detector in the underground facility of Oto. ELEGANTS 23 uses huge mass of $\mathrm{NaI}$ scintillators $(760 \mathrm{Kg}$ ) upgraded from a previous experiment. The background at 
threshold is still high. A search for annual modulation did not show any indication of modulation.

\section{WIMP searches with Xenon Scintilla- tion Detectors}

The search for WIMPs with Xenon scintillators benefits of a well-known technique. Moreover, background discrimination can be done better than in NaI.

One of the pioneer searches using Xenon is the DAMA liquid-Xenon experiment. The spectra of limits on recoils in WIMP- ${ }^{129}$ Xe elastic scattering using PSD and exclusion plots were published in Ref.24. Recent results of the DAMA liquid Xenon experiment refers to limits on WIMP- ${ }^{129}$ Xe inelastic scattering [25]. (See the P.L. Belli contribution to these Proceedings.)

The ZEPLIN Program [29] uses a series of Xenon-based scintillators devices able to discriminate the background from the nuclear recoils in liquid or liquid-gas detectors in various ways. Either using the Scintillation Pulse Shape or measuring the scintillation and the ionization (an electric field prevents recombination, the charge being drifted to create a second scintillation pulse), and capitalizing the fact that the primary (direct) scintillation pulse and the secondary scintillation pulse amplitudes differ for electron recoils and nuclear recoils. The secondary scintillation photons are produced by proportional scintillation process in liquid-Xenon like in the ZEPLIN-I detector, (where a discrimination factor of $98 \%$ is achieved) or by electroluminiscence photons in gas-Xenon (like in the case of the ZEPLIN-II detector prototype) in which the electrons (ionization) are drifted to the gas phase where electroluminiscence takes place (the discrimination factor being $>99 \%$ ) [26]. Some prototypes leave been tested and various different projects of the ZEPLIN series are underway [27,29] to be installed in Boulby. (See S. Hart contribution to these Proceedings).

\section{WIMP searches with Time Projection Chambers}

DRIFT is a detector project sensitive to directionality [28]. It uses a low pressure (10-40 Torr) TPC with Xenon to measure the nuclear recoil track in WIMP-Nucleus interactions. The diffusion constrains the track length observable but DRIFT reduces the diffusion (transversal and longitudinal) using negative ions to drift the ionization instead of drift electrons: gas $\mathrm{CS}_{2}$ is added to capture electrons and so $\mathrm{CS}_{2}^{(-)}$ions are drifted to the avalanche regions (where the electrons are released) for multiwire read-out (no magnetic field needed). The negative ion TPC has a millimetric diffusion an a millimetric track resolution. The proof-of-principle has been performed in miniDRIFTs, where the direction and orientation of nuclear recoils have been seen. The event reconstruction, the measurement of the track length and orientation, the determination of $\mathrm{dE} / \mathrm{dx}$ and the ionization measurement permit a powerful background discrimination (99.9\% gamma rejection and $95 \%$ alpha rejection) leading to a rate sensitivity of $R<10^{-2(-3)} \mathrm{c} / \mathrm{kgday}$. DRIFT will permit to recognize the forward/backward asymmetry and the nuclear recoils angular distribution, which, as already noted, are the most clear distinctive signatures of WIMPs. That will permit hopefully the identification of WIMP. A DRIFT prototype of $1 \mathrm{~m}^{3}$ is under construction [29]. A project of $10 \mathrm{~m}^{3}$ (Xe) scaling up the TPC of $1 \mathrm{~m}^{3}$ (Xe) is under way. (See S. Hart contribution to these Proceedings).

\section{WIMP searches with metastable parti- cle detectors}

WIMP detectors, which use the metastability of the medium where the nuclear targets are embedded, are the (novel) superheated drop detectors (SDD) (like SIMPLE and PICASSO) and the (old) superconducting superheated grains (SSD) (like ORPHEUS). The SDD's consist of a dispersion of droplets $(\oslash \sim 10 \mu \mathrm{m})$ of superheated liquid (freon) in a gel matrix. The energy deposition of a WIMP in the droplets produces a phase transition from the superheated to normal state causing 
vaporization of droplets into bubbles $(\oslash \sim 1 \mathrm{~mm})$, detected acoustically. SSD are essentially intensitive to low LET particles (e, $\gamma, \mu)$, and so good for detecting WIMPs and neutrons. See Ref.[30] for SIMPLE and the contribution of V. Zacek to these Proceedings and [31] for PICASSO.

The Superconducting Superheated Grains (SSG) detectors, like ORPHEUS, is based on the change of phase from the superconducting superheated to the normal state produced by the WIMP energy deposition in micrograins inside a magnetic field, at very low temperatures. The signal is detected through the disappearance of the Meissner effect. The SSG offer good background rejection (97\%) (a single grain is expected to flip per WIMP or nucleon interaction, in contrast to several grains in the case of other particles), and are sensitive to very low energy deposition (as proved in neutron irradiation experiments). An experiment with tin micrograins has just started at the Bern Underground Laboratory (70 m.w.e.). (See the F. Hasenbalg contribution to these Proceedings and Ref.32.34).

\section{WIMP searches with Cryogenic Parti- cle Detectors}

Thermal detectors 33.34 measure the total recoil energy transferred by the WIMP to the nucleus in an absorber, via its small temperature increase (of the order of $\mu K$ if the working temperature is a few $m K$ ) with a suitable sensor. They have quenching factors of about unity $\left(E_{v i s} \sim E_{R}\right)$ and present other advantages over conventional detectors in the search for WIMPs: a better energy resolution (much smaller energy quanta involved in the processes), a very low effective threshold and a wide choice of absorbers or nuclear targets. Moreover, bolometers which also collect charge (or light) can simultaneously measure the phonon and ionization (or scintillation) components of the energy deposition providing a unique tool of background substraction and particle identification.

There exists five cryogenic experiments looking for WIMPs currently running (MIBETA, CRESST, ROSEBUD, CDMS, and EDELWEISS), another one (CUORICINO) being

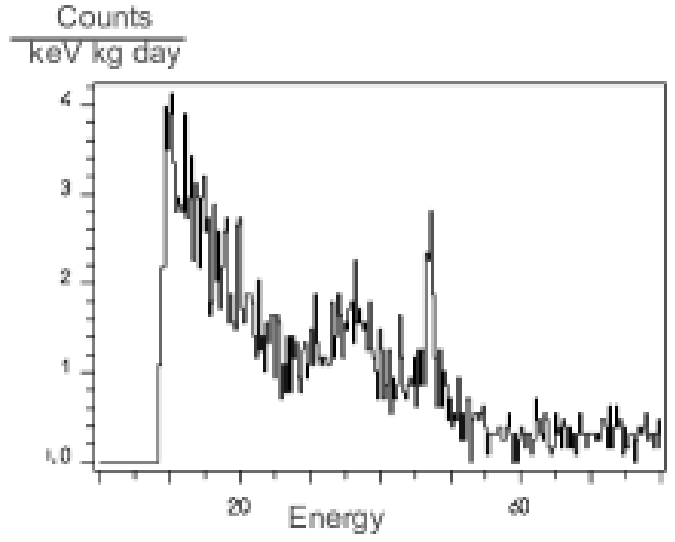

Figure 5.

mounted and a big project (CUORE), in preparation.

The MIBETA experiment 35] consists of an array of 20 bolometers of $\mathrm{TeO}_{2}$ of $340 \mathrm{~g}$ each (the largest cryogenic experiment now in operation), arranged in a tower-like structure running in Gran Sasso. The quenching factor (Q) has been measured to be about 1 (both for Te and O recoils). Although optimized for double beta decay it has produced interesting results in WIMP searches. The low energy background collected with two crystals ( $M=680 \mathrm{~g}$ and $\mathrm{t}=89$ days) with a software threshold of $10 \mathrm{KeV}$ and a hardware threshold of $2-4 \mathrm{KeV}$ is shown in Fig. 5. The exclusion plot derived for such background spectrum gives the best $\sigma$-bound obtained from thermal (only phonons) detectors. (See A. Giulani contribution to these Proceedings.)

CUORE [36] (Cryogenic Underground Observatory for Rare Events) is an experiment projected as a large extension of MIBETA. A first step is CUORICINO, a set of 56 crystals of 760 $\mathrm{g}$ of $\mathrm{TeO}_{2}$ in a tower-structure similar to MIBETA, which is now being mounted. Threshold and energy resolution of the $760 \mathrm{~g}$ detectors are $\sim 5 \mathrm{KeV}$ and $\Gamma(46 \mathrm{KeV})=1 \pm 0.15 \mathrm{KeV}$. In the case of a (flat) background of $10^{-1} \mathrm{c} / \mathrm{KeV} \mathrm{Kg}$ day, which could be reasonably expected extrapolating the current MIBETA results, CUORICINO 


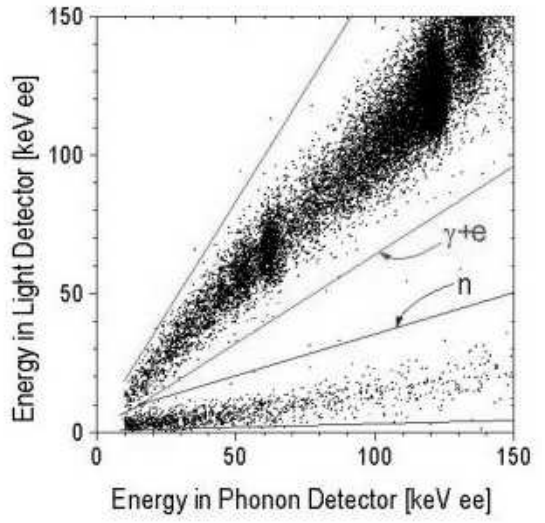

Figure 6.

would permit the exploration of the full DAMA region in $42 \mathrm{Kg}$ year of exposure. (See A. Giulani contribution to these Proceedings and Ref. [37].) The CUORE project will consist of 1000 detectors $(\mathrm{M}=760 \mathrm{Kg})$, forming a cubic structure to be installed in a special dilution refrigerator in the Gran Sasso Laboratory.

The CRESST (Cryogenic Rare Event Search with Superconducting Thermometers) experiment, running also in Gran Sasso, has initiated a new phase which uses scintillating bolometers of calcium tungstate $\left(\mathrm{CaWO}_{4}\right)$ to measure simultaneously the heat and the scintillation produced by the WIMP energy deposit. The calcium tungstate absorbers of $300 \mathrm{~g}$ are viewed by optical bolometers. Fig. 6 shows the discrimination obtained by the CRESST scintillating bolometer, ranging from $99.5 \%$ to $99.7 \%$ above $\sim 20 \mathrm{KeV}$. (See H. Proebst contribution to these Proceedings.) For the Phase I of CRESST, which used thermal (only phonons) detectors of $260 \mathrm{~g}$ of sapphire, we refer to Ref. 38.

ROSEBUD I (Rare Objects Search with Bolometers Underground) is a bolometer experiment currently running in Canfranc. The first phase of the experiment was dedicated to the understanding and reduction of the radioactive background following successive removals of the radioimpure materials. Sapphire $(25 \mathrm{~g}, 50 \mathrm{~g})$ and germanium $(67 \mathrm{~g})$ absorbers of excellent energy resolution were used [39]. Thresholds respectively lower than $1 \mathrm{KeV}$ and $450 \mathrm{eV}$ were achieved on these detectors. Then ROSEBUD has operated simultaneously bolometers of Ge $(67 \mathrm{~g}), \mathrm{Al}_{2} \mathrm{O}_{3}$ $(50 \mathrm{~g})$ and $\mathrm{CaWO}_{4}(54 \mathrm{~g})$. For the first time three different absorbers have been running in the same radioactive environment to help in understanding the background and hopefully permit to investigate the nuclear target dependence of the rate.

The new phase of ROSEBUD will use scintillator bolometers of $\mathrm{CaWO}_{4}(54 \mathrm{~g})$, and of BGO (46 g), to discriminate backgrounds. The scintillating bolometers are mounted facing an small optical bolometer of Ge. Neutron calibrations have shown their high discrimination power. The $\mathrm{CaWO}_{4}$ crystal, tested under neutron irradiation at Orsay, revealed relative light/heat amplitude ratios of $10-2.5-1$ for, respectively, gammas - alphas - recoiling nuclei. Then, in the first underground heat-scintillation discrimination, in Canfranc, no recoil events were observed and an alpha contamination was clearly discriminated from $\gamma / \beta$ background. The discrimination plot of Fig. 7 was recorded (15 hours) in a background running in Canfranc. Threshold was about $45 \mathrm{keV}$ on the heat channel, mainly due to microphonics. The scintillating bolometer of BGO $\left(\mathrm{Bi}_{4} \mathrm{Ge}_{3} \mathrm{O}_{12}\right)(46 \mathrm{~g})$ showed also a high discrimination power in a ${ }^{252} \mathrm{Cf}$ neutron irradiation and in a background running at Canfranc (see Fig. 8). The light/heat factors and the collected light yields $(\approx 0.8 \%$ for gammas $)$ are found very similar to those of $\mathrm{CaWO}_{4}$, while the threshold is much lower for the BGO bolometer, bounded to $6 \mathrm{KeV}$. These features make the BGO bolometers very attractive candidates for a Dark Matter experiment. (See P. de Marcillac contribution to these Proceedings.)

CDMS-I (Cryogenic Dark Matter Search) is a cryogenic experiment installed in the Stanford Underground Facility, which uses Si and Ge hybrid bolometer-diodes, to discriminate nuclear recoils from electron recoils by simultaneous measurement of heat and charge. The rationale is that the ionization Yield (charge/heat), is different for nuclear recoils (WIMPs, n) than for electron recoils (produced by the typical back- 


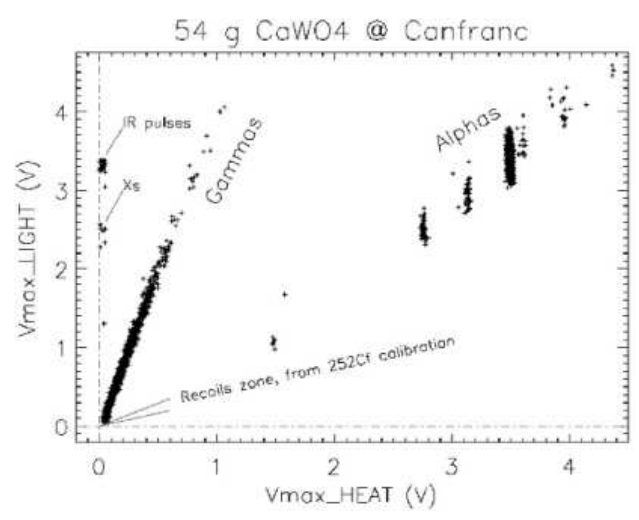

Figure 7.

ground $\gamma, \mathrm{e}, \ldots)$. Typical values of the ionization yield are 1 for electrons bulk events, 0.75 for electrons surface events and 0.3 for nuclear recoil. The electron-hole pairs are efficiently collected in the bulk of the detector, but the trapping sites near the detector surface produce a layer $(10-20 \mu \mathrm{m})$ of partial charge collection, where surface electrons from outside suffer ionization losses and fake nuclear recoils. This is an important limitation of this technique, which recently has been addressed and minimized (95\% discrimination against surface electron background).

Two types of detectors and read-out techniques have been developed: the BLIP germanium detectors where the thermal phonons are read with NTD (Ge) thermistors (in milliseconds). In the ZIP, silicon detectors, athermal phonons are detected (microsecond time scale) with superconducting transition edge thermometers in tungsten. The total deposited energy is measured calorimetrically. The charge is measured as in conventional diodes. The main features and results of CDMS-I have been published in Ref. [15]. An effective exposure of $10.6 \mathrm{Kgday}$, with three BLIP-Ge detectors of $165 \mathrm{~g}$ each, have been used for exclusion plots. The almost perfect discrimination capability of the detector against bulk electron recoils permits to distinguish the nuclear recoil events. A total of 13 nuclear recoil events above $10 \mathrm{KeV}$ can be seen (corresponding to 1.2
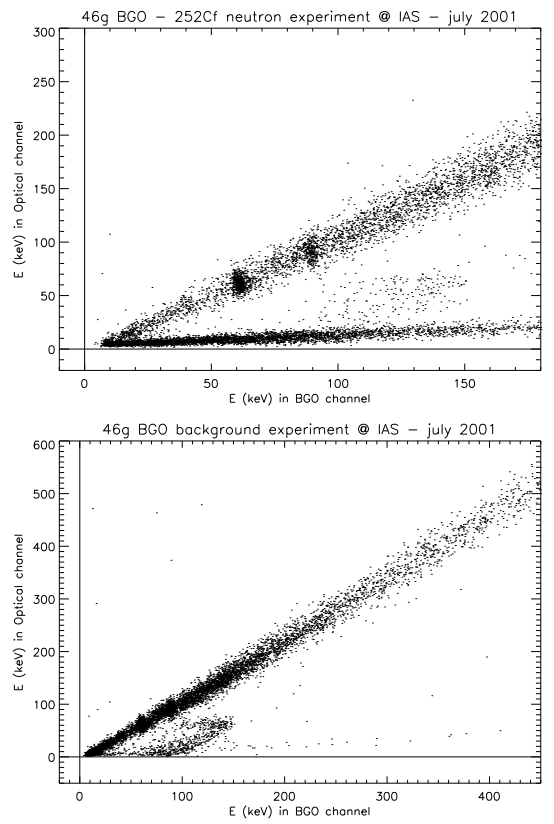

Figure 8.

events/Kgday, see Fig. 9) and are due basically to neutrons. The CDMS events after substraction of the neutron background is shown in Fig. 10, compared with the expected WIMP spectra in Ge for typical $\mathrm{m}, \sigma$ values of the DAMA WIMP. CDMS and DAMA are incompatible at $99.9 \%$ C.L. Exclusion plots obtained from the CDMS data are drawn jointly with that derived from IGEX and EDELWEISS for comparison in Fig. 2, as all three exclude a fraction of the DAMA region (see also B. Sadoulet contribution to these Proceedings).

As the neutrons limits the sensitivity of CDMSI, the next step is to go underground in the Soudan Mine. The objectives are to reduce the neutron background from $1 \mathrm{n} / \mathrm{Kgday}$ down to $10^{-2}-10^{-3} \mathrm{n} / \mathrm{Kgday}$, and to increase substantially the detector mass up to $\sim 7 \mathrm{Kg}(5 \mathrm{Kg}$ of $\mathrm{Ge}$ and $2 \mathrm{Kg}$ of $\mathrm{Si}$ ) with $\mathrm{Ge}$ of $250 \mathrm{~g}$ and $\mathrm{Si}$ of $100 \mathrm{~g}$. The goal of CDMS-II is to decrease the background below $10^{-2}-10^{-3} c / K g d a y$ for $E_{v i s}>10 \mathrm{KeV}$ with the propose of testing S.I. 


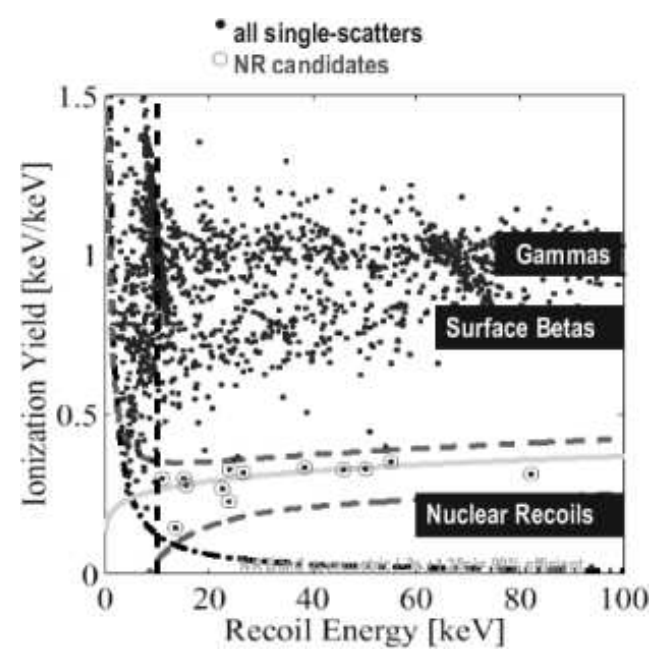

Figure 9.

cross-section interactions neutralino-proton down to $\sim 10^{-8} \mathrm{pb}$, for WIMP masses $50-100 \mathrm{GeVs}$, i.e., well inside the MSSM predictions (see T. Saab contribution to these Proceedings).

EDELWEISS is a cryogenic experiment with Ge hybrid detectors which measure simultaneously ionization and heat [40]. EDELWEISS has operated Ge hybrid bolometers of $70 \mathrm{~g}$ and $320 \mathrm{~g}$ in the Frejus tunnel (LSM) but recent results refer to detectors of $320 \mathrm{~g}(7 \mathrm{~cm} \oslash \times 2 \mathrm{~cm})$. (For the EDELWEISS phase of $2 \times 70 \mathrm{~g}$ Ge detectors see Ref. 40.) The top electrode is divided in a central disk and a guard ring. The preliminary results (2001) show a rate $\mathrm{B}=(20-100) \mathrm{KeV}=1.8 \mathrm{c} / \mathrm{KeV}$ $K g$ day (with the central electrode, $54 \%$ volume). After discrimination, the background turns out to be $\mathrm{B} \sim 0$ in $30-100 \mathrm{KeV}$ : no nuclear recoils were observed in $5.3 \mathrm{Kgday}$ (at the central electrode). That permits to derive an excellent and reliable (without neutron substraction) exclusion plot [16] for spin-independent interaction, which excludes the upper right part of the DAMA region. Fig. 11 shows the physics data takings with the GeAl6 detector $9.31 \mathrm{Kgday}$ (total exposure with $5.3 \mathrm{Kgday}$ from the center electrode). Fig. 12 shows the successive background spec-

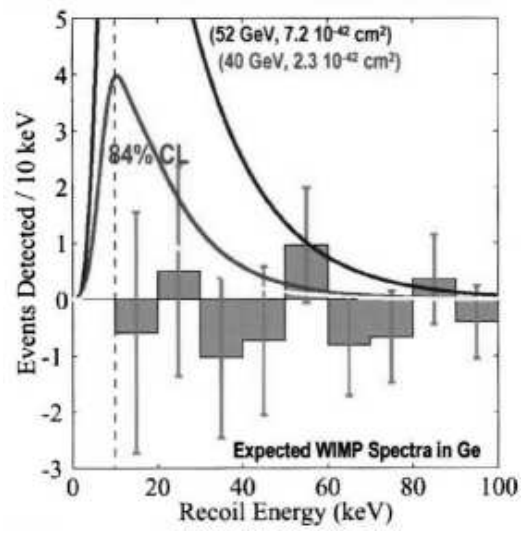

Figure 10.

tra obtained by EDELWEISS with the $70 \mathrm{~g}$ and $320 \mathrm{~g}$ detector. (See O. Martineau contribution to these Proceedings.)

The Collaboration plans to install twenty Ge detectors of $320 \mathrm{~g}$ (in an innovative dilution refrigerator) in the next two-three years, improve the rejection up to $99.99 \%$ and get a background of $10^{-3} \mathrm{c} /(\mathrm{keV} \mathrm{kg}$ day $)$.

\section{Conclusions and outlook}

The direct search for WIMP dark matter proceeds at full strength. More than twenty experiments on direct detection illustrate the effort currently being done. New, dedicated experiments are focusing now in the identification of WIMPs, discriminating the nuclear recoils from the background, rather that in constraining or excluding their parameters space. Their current achievements and the projections of some of them have been reviewed in this talk.

The present experimental situation can be summarized as follows: the rates predicted for SUSY-WIMPs extend from 1-10 c/Kgday down to $10^{-4}-10^{-5} \mathrm{c} /$ Kgday, in scatter plots, obtained within MSSM as basic frame implemented in various alternative schemes. A small fraction of this window is testable by some of the leading experiment. The rates experimentally 


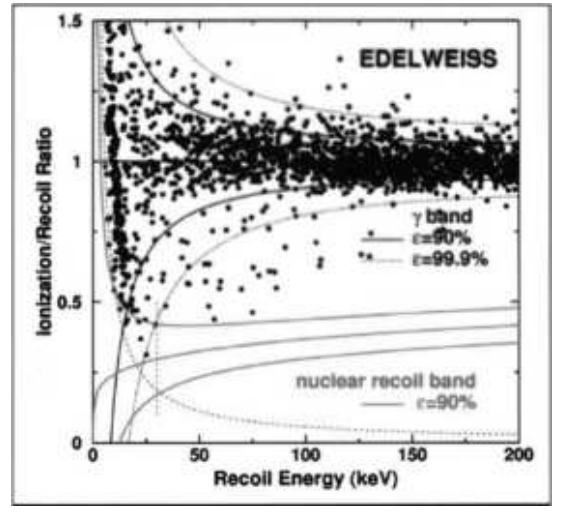

Figure 11.

achieved stand around $1 \mathrm{c} /$ Kgday $(0.1 \mathrm{c} /$ Kgday at hand) (CDMS, EDELWEISS) and differential rates $\sim 0.1-0.05 \mathrm{c} / \mathrm{KeV} \mathrm{Kg}$ day have been obtained by IGEX and $\mathrm{H} / \mathrm{M}$, in the relevant low energy regions. The deepest region of the exclusion plots achieved stands around a few $\times 10^{-6} \mathrm{pb}$, for masses 50-200 GeV (DAMA, CDMS, EDELWEISS, IGEX). The current status of the best exclusion plots is depicted comparatively in Fig. 13. There exists an unequivocal annual modulation effect (see Fig. 1) reported by DAMA (four yearly periods), which has been shown to the compatible (DAMA) with a neutralino-WIMP, of $\mathrm{m} \sim 50-60 \mathrm{GeV}$ and $\sigma_{n}^{S i} \sim 7 \times 10^{-6} \mathrm{pb}$. Recent experiments exclude at greater or lesser extend (CDMS, EDELWEISS, IGEX) the DAMA region.

To reach the lowest rates predicted $\left(10^{-5}\right.$ c/Kgday) in SUSY-WIMP-nucleus interaction, or in other words, to explore coherent interaction cross-sections of the order of $10^{-9}-10^{-10} \mathrm{pb}$, substantial improvements have to be accomplished in pursuing at its best the strategies reviewed in this talk, with special emphasis in discriminating the type of events. These strategies must be focussed in getting a much lower background (intrinsic, environmental, ...) by improving radiopurity and shieldings. The nuclear recoil discrimination efficiency should be optimized going from above $99.7 \%$ up to $99.9 \%$ at the same time that the energy $E_{v i s}$ at which discrimination applies should

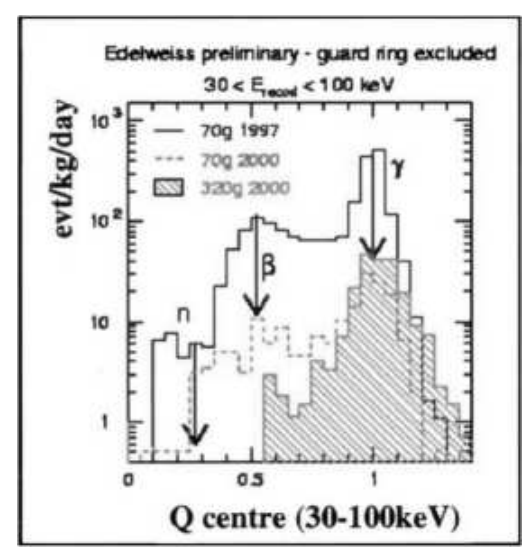

Figure 12.

be lowered. The measurement of the parameters used to discriminate background from nuclear recoils should be improved and finally one needs to increase the target masses and guaranty a superb stability over large exposures. With these purposes various experiments and a large $\mathrm{R}+\mathrm{D}$ activity are under way. Some examples are given in Table 2. The conclusion is that the search for WIMPs is well focused and should be further pursued in the quest for their identification.

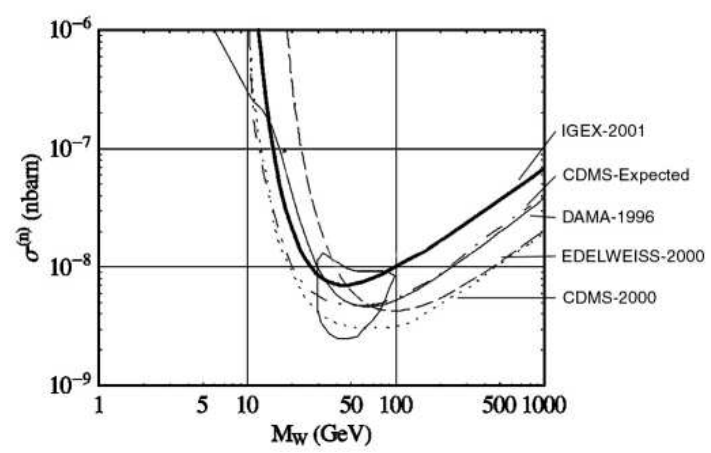

Figure 13. 


\section{Acknowledgments}

I wish to thank S. Cebrián and I.G. Irastorza for their invaluable collaboration in the making of the exclusion plots and to J. Morales for useful discussions. The present work was partially supported by the CICYT and MCyT (Spain) under grant number AEN99-1033 and by the EU Network contract ERB FMRX-CT98-0167.

\section{REFERENCES}

1. A.K. Drukier et al., Phys. Rev. D33 (1986) 3495.

2. D. N. Spergel, Phys. Rev. D 37 (1988) 1953.

3. J. R. Primack, D. Seckel and B. Sadoulet, Ann. Rev. Nucl. Part. Sci. 38 (1988) 751. Jungman, M. Kamionkowski and K. Griest, Phys. Rep. 267 (1996) 195.

4. A.Morales. Review talk at the TAUP'99 Workshop. Nucl. Phys. B (Proc. Suppl.) 87 (2000) 477.

5. A. Morales, Proc. Neutrino Telescopes Workshop, Venice, February 1999, p. 24. Y. Ramachers, XI Rencontres de Blois, June 1999, astro-ph/9911260. L. Baudis and H.V. Klapdor, lastro-ph/0003434.

6. A.Bottino et al. DFTT 35/2000 and references therein. J. Ellis et al. hep-ph/0007113 and P. Gondolo hep-ph/0008022.

7. P. F. Smith and J. D. Lewin. Phys. Rep. 187 (1990) 203.

8. J. Copi, J. Heo and L. M. Krauss, Phys. Lett. B 461 (1999) 43.

9. A. Morales, Proc. of South Carolina Symposium on Neutrino Physics, Ed. H. Kuborera et al., World Scietific Pub. 2000. A. Morales, Invited talk given at the 29th International Meeting on Fundamental Physics, Sitges (Barcelona), Feb. 2001, to be published in the Proceedings, hep-ex/0111089].

10. C. Aalseth et al., Phys. Rev. C59 (1999) 2108.

11. A. Morales et al. [IGEX Collaboration], Phys. Lett. B 489 (2000) 268 hep-ex/0002053].

12. A. Morales et al. [IGEX Collaboration], hep$\mathrm{ex} / 0110061$.

13. L. Baudis et al., Phys. Rev. D59 (1999)
022001.

14. R. Bernabei et al., Phys. Lett. B450 (1999) 448 and Phys. Lett. B480 (2000) 23.

15. R. Abusaidi et al. [CDMS Collaboration], Phys. Rev. Lett. 84 (2000) 5699 astro$\mathrm{ph} / 0002471$.

16. A. Benoit et al. [EDELWEISS Collaboration], Phys. Lett. B513 (2001) 15 astro$\mathrm{ph} / 0106094$.

17. R. Bernabei et al, Phys. Lett. B379(1996)299.

18. L. Baudis et al., Phys. Rev. D 63 (2000) 022001.

19. L. Baudis et al., hep-ex/0012022.

20. S. Cebrián et al., these Proceedings.

21. P. F. Smith et al., Phys. Lett. B 379 (1996) 299 and N. J. C Spooner et al., Phys. Lett. B 473 (2000) 330.

22. G. Gerbier et al., Astrop. Phys. 11 (1999) 287.

23. K. Fushimi et al., Astrop. Phys. 12 (2000) 185.

24. R. Bernabei et al., Phys. Lett. B 436 (1998) 379.

25. R. Bernabei et al., NJP 2 (2000) 15.1.

26. R. Luscher et al., Nucl. Phys. B (Proc. Suppl.) 95 (2001) 233.

27. D.Cline, ZEPLIN-IV. Contribution to the Snowmass2001 Workshop, astro$\mathrm{ph} / 0108045$.

28. C. J. Martoff et al., Nucl. Instr. and Meth. in Phys. Res. A 440 (2000) 355.

29. N. J. C. Spooner, The Future of Particle Physics, Snowmass 2001.

30. J. I. Collar et al., NJP 2 (2000) 14.

31. V. Zacek, Nucl. Phys. B (Proc. Suppl.) 91 (2001) 368.

32. B. van den Brandt et al., Nucl. Phys. B (Proc. Suppl.) 87 (2000) 117.

33. E. Fiorini and T. O. Niinikoski, Nucl. Intr. and Meth. in Phys. Res. A 224 (1984) 83.

34. K. Pretzl, Nucl. Instr. and Meth. in Phys. Res. A 454 (2000) 114.

35. A. Alessandrello et al., Phys. Lett. B 486 (2000) 13.

36. E. Fiorini, Phys. Rep. 307 (1998) 309. Letter of intent to the Gran Sasso Scientific Committee and to the Funding Authorities, March, 2001.

37. I. G. Irastorza et al., Aug. 2001, hep- 
$\mathrm{ph} / 0108146]$.

38. J. Jochum et al., Nucl. Phys. B. (Proc. Suppl.) 87 (2000) 70.

39. S. Cebrián et al., Astrop. Phys. 10 (1999) 361 and Astrop. Phys. 15 (2001) 79.

40. G. Chardin et al., Nucl. Phys. B (Proc. Suppl.) 87 (2000) 74 . 
Table 1

WIMP Direct Detection in underground facilities experiments currently running (or in preparation)

\begin{tabular}{|c|c|c|}
\hline LABORATORY & EXPERIMENT & TECHNIQUE \\
\hline BAKSAN (Russia) & IGEX & $3 \times 1 \mathrm{Kg}$ Ge-ionization \\
\hline BERN(Switzerland) & ORPHEUS & (SSD) Superconducting Superheated Detector, $0.45 \mathrm{Kg}$ Tin \\
\hline BOULBY & $\mathrm{NaI}$ & NaI scintillators of few Kg (recently completed) \\
\hline \multirow[t]{3}{*}{$(\mathrm{UK})$} & NAIAD & NaI unencapsulated scintillators (50 Kg) \\
\hline & ZEPLIN & $\begin{array}{l}\text { Liquid-Gas Xe scintillation/ionization I: } 4 \text { Kg single phase } \\
\text { II: } 30 \mathrm{Kg} \text { Two phases }\end{array}$ \\
\hline & DRIFT & Low pressure Xe TPC (in preparation) $1 \mathrm{~m}^{3} \rightarrow 10 \mathrm{~m}^{3}$ \\
\hline CANFRANC & COSME & $234 \mathrm{~g}$ Ge ionization \\
\hline \multirow{4}{*}{ (Spain) } & IGEX & $2.1 \mathrm{Kg}$ Ge ionization \\
\hline & ANAIS & $10 \times 10.7 \mathrm{Kg}$ NaI scintillators \\
\hline & ROSEBUD & $50 \mathrm{~g} \mathrm{Al}_{2} \mathrm{O}_{3}$ and $67 \mathrm{~g}$ Ge thermal detectors \\
\hline & & $\mathrm{CaWO}_{4} 54 \mathrm{~g}$ and $\mathrm{BGO} 46 \mathrm{~g}$ scintillating bolometers \\
\hline FREJUS/MODANE & SACLAY-NaI & 9.7 Kg NaI scintillator (recently completed) \\
\hline \multirow{2}{*}{ (France) } & EDELWEISS I & $70 \mathrm{~g}$ Ge thermal+ionization detector \\
\hline & EDELWEISS II & $4 \times 320$ g Ge thermal+ionization detectors \\
\hline GRAN SASSO & $\mathrm{H} / \mathrm{M}$ & 2.7 Kg Ge ionization \\
\hline \multirow{11}{*}{ (Italy) } & HDMS & 200g Ge ionization in Ge well \\
\hline & GENIUS-TF & $40 \times 2.5 \mathrm{Kg}$ unencapsulated Ge (in preparation) \\
\hline & DAMA & NaI scintillators $(87.3 \mathrm{Kg})$ \\
\hline & LIBRA & NaI scintillators $250 \mathrm{Kg}$ (in preparation) \\
\hline & Liquid-Xe & Liquid Xe scintillator $(6 \mathrm{Kg})$ \\
\hline & $\mathrm{CaF}_{2}$ & Scintillator \\
\hline & CRESST I & $(4 \times 260 \mathrm{~g}) \mathrm{Al}_{2} \mathrm{O}_{3}$ thermal detectors \\
\hline & CRESST II & Set of $300 \mathrm{~g} \mathrm{CaWO}_{4}$ scintillating bolometers (up to $10 \mathrm{Kg}$ ) \\
\hline & MIBETA & $20 \times 340 \mathrm{~g} \mathrm{TeO}_{2}$ thermal detector \\
\hline & CUORICINO & $56 \times 760 \mathrm{~g} \mathrm{TeO}{ }_{2}$ thermal detector (being mounted) \\
\hline & CUORE & $1000 \times 760 \mathrm{~g} \mathrm{TeO}_{2}$ (in preparation) \\
\hline RUSTREL (France) & SIMPLE & (SDD)Superheated Droplets Detectors (Freon) \\
\hline STANFORD UF/ & CDMS - I & $100 \mathrm{~g} \mathrm{Si} ; 6 \times 165 \mathrm{~g}$ Ge thermal+ionization detectors \\
\hline SOUDAN(USA) & CDMS - II & $3 \times 250 \mathrm{~g}$ Ge and $3 \times 100 \mathrm{~g}$ Si Thermal+Ionization \\
\hline SNO (Canada) & PICASSO & (SDD)Superheated Droplets Detectors (1.34g of Freon) \\
\hline OTO & ELEGANTS-V & Large set of massive $\mathrm{NaI}$ scintillators \\
\hline (Japan) & ELEGANTS-VI & $\mathrm{CaF}_{2}$ scintillators \\
\hline
\end{tabular}


Table 2

WIMP Direct Detection Prospect

\begin{tabular}{|c|c|}
\hline & BEING INSTALLED/OR PHASE II EXPERIMENTS (To start 2001-2002) \\
\hline CDMS-II & $(\mathrm{Ge}, \mathrm{Si})$ Phonons+Ioniz $7 \mathrm{Kg}, \mathrm{B} \sim 10^{-2}-10^{-3} \mathrm{c} / \mathrm{Kgd}, \sigma \sim 10^{-8} \mathrm{pb}$ \\
\hline EDELWEISS-II & (Ge) Phonons+Ioniz $6.7 \mathrm{Kg}, \mathrm{B} \sim 10^{-2}-10^{-3} \mathrm{c} / \mathrm{Kgd}, \sigma \sim 10^{-8} \mathrm{pb}(40-200 \mathrm{GeV})$ \\
\hline CUORICINO & $\mathrm{TeO}_{2}$ Phonons $42 \mathrm{Kg}, \mathrm{B} \sim 10^{-2} \mathrm{dru}, \sigma \sim 0^{-7} \mathrm{pb}$ \\
\hline CRESST-II & $\begin{array}{l}\mathrm{CaWO}_{4} \text { Phonons+light, } B<10^{-2}-10^{-3} \text { dru }(15 \mathrm{KeV}), \sigma \sim 10^{-7}-10^{-8} \mathrm{pb} \\
(50-150 \mathrm{GeV})\end{array}$ \\
\hline IGEX & Ge Ioniz $2.1 \mathrm{Kg}, \mathrm{B}<10^{-1}-10^{-2} \mathrm{dru}, \sigma \sim 2 \times 10^{-6} \mathrm{pb}(40-200 \mathrm{GeV})$ \\
\hline HDMS & Ge Ioniz $0.2 \mathrm{Kg}, \sigma \sim 6 \times 10^{-6} \mathrm{pb}(20-80 \mathrm{GeV})$ \\
\hline ANAIS & NaI Scintillators $107-150 \mathrm{Kg}, \mathrm{B}(\mathrm{PSD}) \leq 0.1 \mathrm{dru}, \sigma \sim 2 \times 10^{-6} \mathrm{pb}$ \\
\hline \multirow[t]{2}{*}{ NAIAD } & NaI Scintillators $10-50 \mathrm{Kg}, \mathrm{B}(\mathrm{PSD}) \leq 0.1 \mathrm{dru}, \sigma \sim 10^{-6} \mathrm{pb}(60-200 \mathrm{GeV})$ \\
\hline & IN PREPARATION (To start 2002-2003) \\
\hline LIBRA (DAMA) & NaI Scintillators $250 \mathrm{Kg}$ \\
\hline GENIUS-TF & $\begin{array}{l}\text { Ge Ioiniz } 40 \mathrm{Kg}, \mathrm{B}<10^{-2} \mathrm{dru}, E_{T h r}=10 \mathrm{KeV} \rightarrow \sigma \sim 10^{-6} \mathrm{pb}(40-200 \mathrm{GeV}) \text {, } \\
E_{T h r}=2 \mathrm{KeV} \rightarrow \sigma \sim 10^{-7} p b(20-80 \mathrm{GeV})\end{array}$ \\
\hline ZEPLIN-II & Xe-Two-phase $40 \mathrm{Kg}$, NR discrim $>99 \%, \mathrm{~B}<10^{-2}$ dru, $\sigma \sim 10^{-7} \mathrm{pb}$ \\
\hline \multirow[t]{2}{*}{ DRIFT-I } & Xe TPC $1 \mathrm{~m}^{3}, \mathrm{~B}<10^{-2}$ dru, $\sigma \sim 10^{-6} \mathrm{pb}(80-120 \mathrm{GeV})$ \\
\hline & IN PROJECT (>2003-2005) \\
\hline CUORE & $\mathrm{TeO}_{2}$ Phonons $760 \mathrm{Kg}, E_{T h r} \sim 2.5 \mathrm{KeV}, \mathrm{B} \sim 10^{-2}-10^{-3}$ dru, $\sigma \sim 5 \times 10^{-8} \mathrm{pb}$ \\
\hline GENIUS 100 & Ge ioniz $100 \mathrm{Kg}, E_{T h r} \sim 10 \mathrm{KeV}$ \\
\hline (GENINO) & $\mathrm{B} \sim 10^{-3}-10^{-5} \mathrm{dru}, \sigma \sim 5 \times 10^{-8}-2 \times 10^{-9} \mathrm{pb}$ \\
\hline \multirow[t]{2}{*}{ GEDEON } & Ge ioniz $28-112 \mathrm{Kg}, \mathrm{B} \sim 2 \times 10^{-3} \mathrm{dru}(>10 \mathrm{KeV}) \sigma \sim 10^{-7}-10^{-8} \mathrm{pb}(40-200 \mathrm{GeV})$ \\
\hline & THE FUTURE (>2005-2007) \\
\hline DRIFT 10 & Xe $10 \mathrm{~m}^{3}$ TPC, $\sigma \sim 10^{-} 8 \mathrm{pb}$ \\
\hline ZEPLIN-MAX & Xe Two-Phase, $\sigma \sim 10^{-10} \mathrm{pb}$ \\
\hline GENIUS & Ge ioniz 1-10 Tons, $\sigma \sim 10^{-9}-10^{-10} \mathrm{pb}$ \\
\hline DRIFT-1 ton & Xe 1 Ton TPC, $\sigma \sim 10^{-10}-10^{-11} \mathrm{pb}$ \\
\hline
\end{tabular}

